\title{
Design of LED streetlight control system based on the Zigbee wireless network
}

\author{
Xiaguang Dong ${ }^{1, a}$, Wang Bin $^{2, b}$ \\ ${ }^{1}$ Institute of Electrical and Information Engineering, Anhui University of Science and Technology, \\ Huainan 232001,China \\ ${ }^{2}$ Institute of Electrical and Information Engineering, Anhui University of Science and Technology, \\ Huainan 232001,China \\ aEmail:328500425@qq.com, ${ }^{\text {b } E m a i l: ~ 285277712 @ q q . c o m ~}$
}

Keywords: ZigBee technology; CC2530; GPRS; street light control system

\begin{abstract}
With the development of our economy and the improvement of urbanization level, lighting system has increased largely and significantly. So energy saving lamp is significant to some extent. This paper has designed a kind of street light control system based on ZigBee wireless network. Using this control system, the state information of every street light are sent to the network gateway, which collects the information transmitted to the monitor system centre by GPRS network, the monitor system can control and inspect every street light.
\end{abstract}

\section{Introduction}

In recent years, wireless communication technology is developing rapidly and meanwhile is widely used in monitoring and control field. To support the sutainable development of economy, saving energy and improving the utilization rate of street lamps has become an urgent problem needed to be solved. Combining with all kinds of sensors and power controllers, ZigBee technology, with its own characteristics of both-way communication, low cost, low power consumption and large capacity of the net, has saved resource. And the technology provides a more reasonable solution for the street light automatic control field. This paper mainly illustrates the software and the hardware design of related part of the ZigBee street light control system.

\section{An introduction to ZigBee technology}

ZigBee is a wireless network technology based on IEEE802.15.4 standard. Generally, with the character of short distance, low complexity, low power consumption, low data rate and low cost, ZigBee is commonly used in electric devices which are short distance and low requirements for transmission rate to achieve coordination among communications in a number of sensors. In the network layer, star network and network topology can be used. Nodes can be divided into Coordinator, Router and FFD according to its function. Besides what mentioned above, ZigBee also has the advantages of high reliability and large network capacity.

Zigbee street control system can be divided into three parts: the monitoring centre, gateway and subnet ZigBee. The system realizes remote monitoring through the ZigBee network and GPRS network connectivity. The system frame is shown in figure 1.

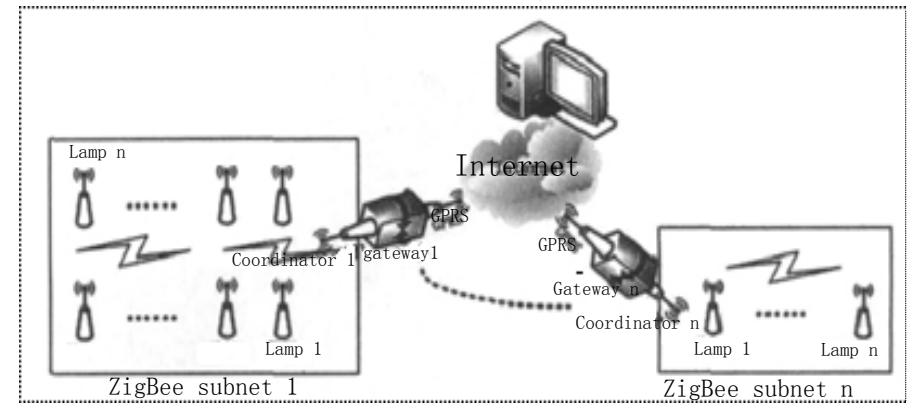

Fig.1. The control system of zigbee street light 
ZigBee nodes have been installed on LED lights according to the communication protocol network after all the ZigBee coordinators have finished network construction. The message on LED nodes will be transmitted to the coordinator, then to the GPRS network, and then to monitoring by Internet. On the one hand, the monitoring centre can send the control or monitoring instruction to ZigBee subnet for control and detection of every street lamp. On the other hand, the fault information of nodes and subnet in ZigBee network will be send to the monitor contre. That will form a two-way communication link.

\section{Hardware design of system}

ZigBee module adopts CC2530 on-ship system as the core of control circuit, with 256KB FLASH and the current loss less than $25 \mathrm{~mA}$ and $34 \mathrm{~mA}$ in receiving and transmitting mode. VCC is provided $5 \mathrm{~V}$ voltage by external regulated power to ensure that all the nodes work stably for a long time.

Based on the characteristics of the street lamp system, the network connected by a tree network, so that extension nodes and the Ad hoc network are more flexible. As the network coordinator, ZigBee module connecting to the PC serial port RS232 plays the role of a organization, network management and orders. When some nodes join the network, ZigBee module will distribute of address to a new node, therefore it cannot be in the state of power down and low power. ZigBee module, connected with street lamps, plays a role of Router, can send and receive data, undertake the task of communicating with the PC and the adjacent nodes.

Street lamp node consists of street lamp controller, power supply module and ZigBee module , finishes processing the one-site data and the control command, complete the task of LED lamp switch and dimming.

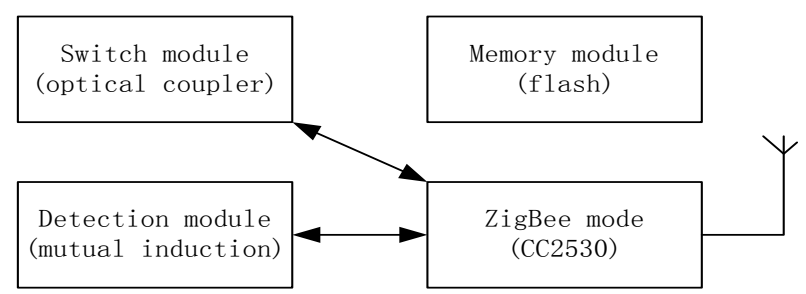

Fig.2. Street lamp node diagram

8 bit AVR microprocessor which is high performance and low consumption is chosen as core component. This SCM combines a variety of devices and functions in one, greatly reduces the usage of peripheral devices and the complexity of the circuit.

Signal detection part is made up of photosensitive resistance circuit and pyroelectric infrared sensor circuit. Photosensitive resistance, named 5537, is used to detect ambient light to control the brightness of the light.

The monitoring centre, composed of PC and ZigBee module, provides system information, has the man-made interface display and can monitor the whole system in real time.

PC can collect information of street lights' condition such as environment light intensity, electricity, light, etc, by wireless communication network. Besides, it can send control commands to the street lamp node. According to the commands received, street lamp node will manipulate LED.

\section{Software design of system}

A gateway is realized by the main control chip LPC1227 which controls ZigBee module and GPRS module and mainly solve the problem of communication. The realization form of GPRS wireless communication module is receiving and sending SMS, connecting GPRS network, etc. The establishment of ZigBee wireless network, data collection and control of other functions are implemented by transmitting a serial port instruction.

The process of establishing network ZigBee network is the process of ZigBee module initialization. ZigBee module is the coordinator on the gateway. After establishment network successfully, coordinator is in the state of waiting for node access. When the same channel and 
routing or terminal of PANID apply for the network, the coordinator will agree the application and save the information from node at the same time. When received the order, the coordinator will transmit the order to ZigBee network.

ZigBee module's is to communicate with the gateway node. Depending on the role, ZigBee module of street lamp node is divided into terminal and routing module during the process of communication. If it is terminal module, this street light node will communicate with the gateway node directly. Else this street light node will transmit other terminal or routing data.

According to the actual operation situation of street lamp, if there are fewer vehicles and pedestrians on the road, turn off part of the lamps. Else, make all the street lights in the state of on. So the state of light can be controlled according to different periods of time. Firstly, one day is divided into daytime(7:00-19:00), night(19:00-01:00) and before dawn(01:00-07:00). Secondly, all the street lights are divided into odd number street lamp working mode, even number street lamp working mode and all number street lamp working mode according to the node number. Finally, LED street light is controlled to ensure road safety and energy saving effect according to the different time periods and different path segments. The operation mode of the street lamp is shown in table 1.

Table 1

\begin{tabular}{cc}
\hline Periods of time & Working mode \\
\hline Daytime & Close all street lamps \\
Night & Open all street lamps \\
Dawn & Open odd number lamps \\
\hline
\end{tabular}

Parameters of time and working mode above is send to all the nodes of ZigBee network by the gateway. And then nodes constantly query the RTC clock and compare the parameters to switch the work mode. This way can be easily changed according to the practical application environment. The flow chart is shown in figure 3.

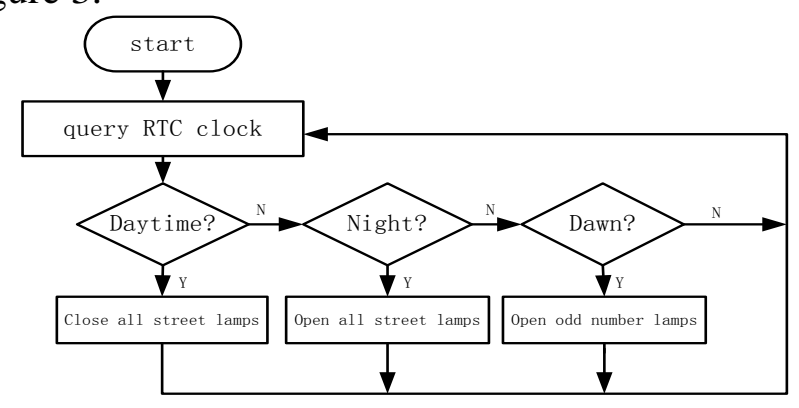

\section{Conclusion}

Fig.3. Energy saving control flow chart

The system can control area street lights by the ZigBee wireless communication technology and have strong practicability. Manage easily, basically solve the maintenance problem and promote the rapid development of the theory of low carbon economy to some extent. Urban lighting will be development direction in a period time of future.

\section{References}

[1]Wang Hao. Discussion on how to make solar LED lighting develop with urban construction harmoniously[J]. China Illuminating Engineering Journal, 2012, 23(2), 33-35.

[2]FARAHANI S.ZigBee wireless network and transceivers [M]. Newnes, 2008.

[3]Zhaobing Chu. CITY LIGHTING WIRELESS CONTROL SYSTEM BASED ON CPRS [D].Shanghai: Shanghai Jiaotong University, 2009.

[4]Yang Xiaoquan. Range Extender ZigBee Gateway Based on LPC1227 [J]. Microcontroller and embedded system applications, 2011(11), 33-66.

[5]Gao Shouwei, Wu Canyang. ZigBee Technology Practice Guide[M]. Beijing: Beijing University of Aeronautics and Astronautics, 2008. 